\title{
Identification of Potential Transcriptomic Markers in Developing Ankylosing Spondylitis: A Meta-Analysis of Gene Expression Profiles
}

\author{
Fang Fang, Jian Pan, Lixiao Xu, Gang Li, and Jian Wang \\ Institute of Pediatric Research, Children's Hospital of Soochow University, Suzhou 215003, China \\ Correspondence should be addressed to Jian Wang; wj196312@vip.163.com
}

Received 10 June 2014; Accepted 27 October 2014

Academic Editor: Jozef Anné

Copyright (c) 2015 Fang Fang et al. This is an open access article distributed under the Creative Commons Attribution License, which permits unrestricted use, distribution, and reproduction in any medium, provided the original work is properly cited.

The goal of this study was to identify potential transcriptomic markers in developing ankylosing spondylitis by a meta-analysis of multiple public microarray datasets. Using the INMEX (integrative meta-analysis of expression data) program, we performed the meta-analysis to identify consistently differentially expressed (DE) genes in ankylosing spondylitis and further performed functional interpretation (gene ontology analysis and pathway analysis) of the DE genes identified in the meta-analysis. Three microarray datasets (26 cases and 29 controls in total) were collected for meta-analysis. 905 consistently DE genes were identified in ankylosing spondylitis, among which 482 genes were upregulated and 423 genes were downregulated. The upregulated gene with the smallest combined rank product $(\mathrm{RP})$ was GNG11 (combined RP $=299.64$ ). The downregulated gene with the smallest combined RP was S100P (combined RP = 335.94). In the gene ontology $(\mathrm{GO})$ analysis, the most significantly enriched $\mathrm{GO}$ term was "immune system process" $\left(P=3.46 \times 10^{-26}\right)$. The most significant pathway identified in the pathway analysis was antigen processing and presentation $\left(P=8.40 \times 10^{-5}\right)$. The consistently DE genes in ankylosing spondylitis and biological pathways associated with those DE genes identified provide valuable information for studying the pathophysiology of ankylosing spondylitis.

\section{Introduction}

Ankylosing spondylitis (AS) represents a chronic inflammatory arthritis, which affects the axial joints such as spine and sacroiliac joints [1]. It causes serious spinal mobility impairment and influences the quality of life [2]. Ankylosing spondylitis is a complex and systemic rheumatic disease; hence systematic screening is required to improve the diagnosis and treatment of ankylosing spondylitis.

Rapid growth of high-throughput transcriptomic data largely enables gene expression profiling and diagnostic targets identification in disease nowadays. In the past decade, several studies have focused on the transcriptional profiling of ankylosing spondylitis using microarrays to identify candidate genes involved in ankylosing spondylitis [3, 4]. Analysis of multiple transcriptomic datasets has the likelihood of discovering robust candidates for diagnosis and treatment. Therefore, we investigated gene expression patterns between ankylosing spondylitis patients and healthy controls in a meta-analysis based on public microarray datasets. The differently expressed genes identified in the meta-analysis were further interpreted by gene ontology analysis and pathway analysis.

To carry out these studies, we used the INMEX (integrative meta-analysis of expression data) program [5]. Careful data procession and annotation were done to insure that the data format and class labels were consistent across datasets. Due to the differences in study design and platform usage, heterogeneity exists among microarray datasets. To address this, we applied the combing rank orders algorithm based on the RankProd package [6], which is robust facing outliers and variations among studies, to carry out the meta-analysis.

\section{Materials and Methods}

2.1. Microarray Datasets Search and Selection. In this study, we searched public microarray study till March 18, 2014, according to the keywords "ankylosing spondylitis" in 
TABLE 1: Studies and data included in this meta-analysis.

\begin{tabular}{lccccc}
\hline \multirow{2}{*}{ Study } & \multirow{2}{*}{ GEO accession } & \multicolumn{2}{c}{ Sample size } & \multirow{2}{*}{ Sample source } & \multirow{2}{*}{ Platform } \\
& & AS case & Control & & \\
\hline 1 & GSE41038 & 2 & 4 & Synovial biopsies & GPL6883 Illumina HumanRef-8 v3.0 expression beadchip \\
2 & GSE25101 & 16 & 16 & Blood & GPL6947 Illumina HumanHT-12 V3.0 expression beadchip \\
3 & GSE11886 & 8 & 9 & Blood & GPL570 Affymetrix Human Genome U133 Plus 2.0 Array \\
\hline
\end{tabular}

GEO: Gene Expression Omnibus; AS: ankylosing spondylitis.

Gene Expression Omnibus (GEO) database (http://www.ncbi .nlm.nih.gov/geo/) [7]. The studies obtained were fur ther selected for the meta-analysis and our selection criteria were (a) case-control study; (b) study providing gene expression data; and (c) study with ankylosing spondylitis patients diagnosed based on the modified New York criteria [8]. Animal studies and studies not about ankylosing spondylitis were excluded in this meta-analysis.

Two investigators independently collected data from each eligible study. The data were composed of GEO accession, sample size, sample source, platform, and gene expression data. Through checking between the two investigators, a final data collection was determined.

2.2. Meta-Analysis Methods. According to the data collected from each eligible microarray study, we performed an overall meta-analysis to identify differentially expressed (DE) genes in ankylosing spondylitis. In this study, we used the INMEX (integrative meta-analysis of expression data) program (http://www.inmex.ca/INMEX/) [5] to carry out the meta-analysis.

All eligible datasets were uploaded to INMEX, then processed, and annotated to insure that the data format and class labels were consistent across datasets. After data integrity check, we carried out a meta-analysis using combing rank orders algorithm, with 100 times of permutation tests. The combing rank order algorithm is based on the RankProd package [6] and is robust facing outliers and variations among studies.

2.3. Functional Interpretation Methods. Functional interpretation (gene ontology analysis and pathway analysis) of the DE genes identified in the meta-analysis was further performed using the INMEX program. In gene ontology (GO) analysis, a $P$ value threshold of 0.05 was used to identify significantly enriched GO terms [9]. In pathway analysis, enrichment analysis was carried out using the hypergeometric test with a $P$ value threshold of 0.05 based on the KEGG database [10].

\section{Results}

3.1. Studies and Data Included in This Meta-Analysis. Original search identified 8 studies in total. Then, 5 studies were excluded among which 4 were not about the DE genes between ankylosing spondylitis patients and healthy controls, and 1 was animal study. Through searching and selection, a final list of 3 microarray datasets $[3,4]$ was collected for meta-analysis. In total, the 3 eligible datasets consisted of 26 cases and 29 controls. All 3 datasets provided casecontrol data with various sample sources ( 1 dataset of synovial biopsies sample and 2 datasets of blood sample). The detailed information of these 3 datasets is presented in Table 1. Heat map of rescaled individual expression data for a subset of genes across the 3 datasets is shown in Figure 1, and the patterns of change for a gene among different datasets could be visualized.

3.2. Meta-Analysis Results. In this study we performed the meta-analysis based on combing rank orders, and the DE genes with $P$ value $<0.05$ were selected. Overall, there were 743 gained genes and 167 lost genes in this metaanalysis (Figure 2, see Supplementary Table 1 in Supplementary Materials available online at http://dx.doi.org/10.1155/ 2014/826316). Gain genes are those identified to be differentially expressed uniquely in the meta-analysis. The expression profiles of gain genes are relatively weak but consistent across datasets. They benefit by larger sample size and hence are more reliable DE genes. Lost genes are those identified to be differentially expressed in individual analysis rather than in the meta-analysis. The expression profiles of lost genes vary largely across different datasets [5].

In total, according to the results of our meta-analysis, 905 genes were identified to be differentially expressed between ankylosing spondylitis patients and healthy controls across microarray datasets (Supplementary Table 2). Among the 905 DE genes, 482 genes were upregulated and 423 genes were downregulated. The top 10 most significantly upregulated genes and top 10 most significantly downregulated genes are shown in Table 2. The upregulated gene with the smallest combined rank product (RP) was GNG11 (combined RP = 299.64). GNG11, guanine nucleotide binding protein $(\mathrm{G}$ protein) gamma 11, is a member of the $G$ protein gamma family which functions in the transmembrane signaling system and cellular senescence [11, 12]. The downregulated gene with the smallest combined RP was S100P (combined RP $=335.94)$. $\mathrm{S} 100 \mathrm{P}(\mathrm{S} 100$ calcium binding protein $\mathrm{P})$ belongs to the S100 calcium-binding protein family and functions in the regulation of diverse cellular processes [13]. However, neither GNG11 nor S100P have been reported to be associated with ankylosing spondylitis yet.

Many consistently DE genes across datasets identified are involved in immune regulation, such as COMMD6, C19orf59, CCR7, CX3CR1, CFD, and FGFBP2 (see Table 2). Although 


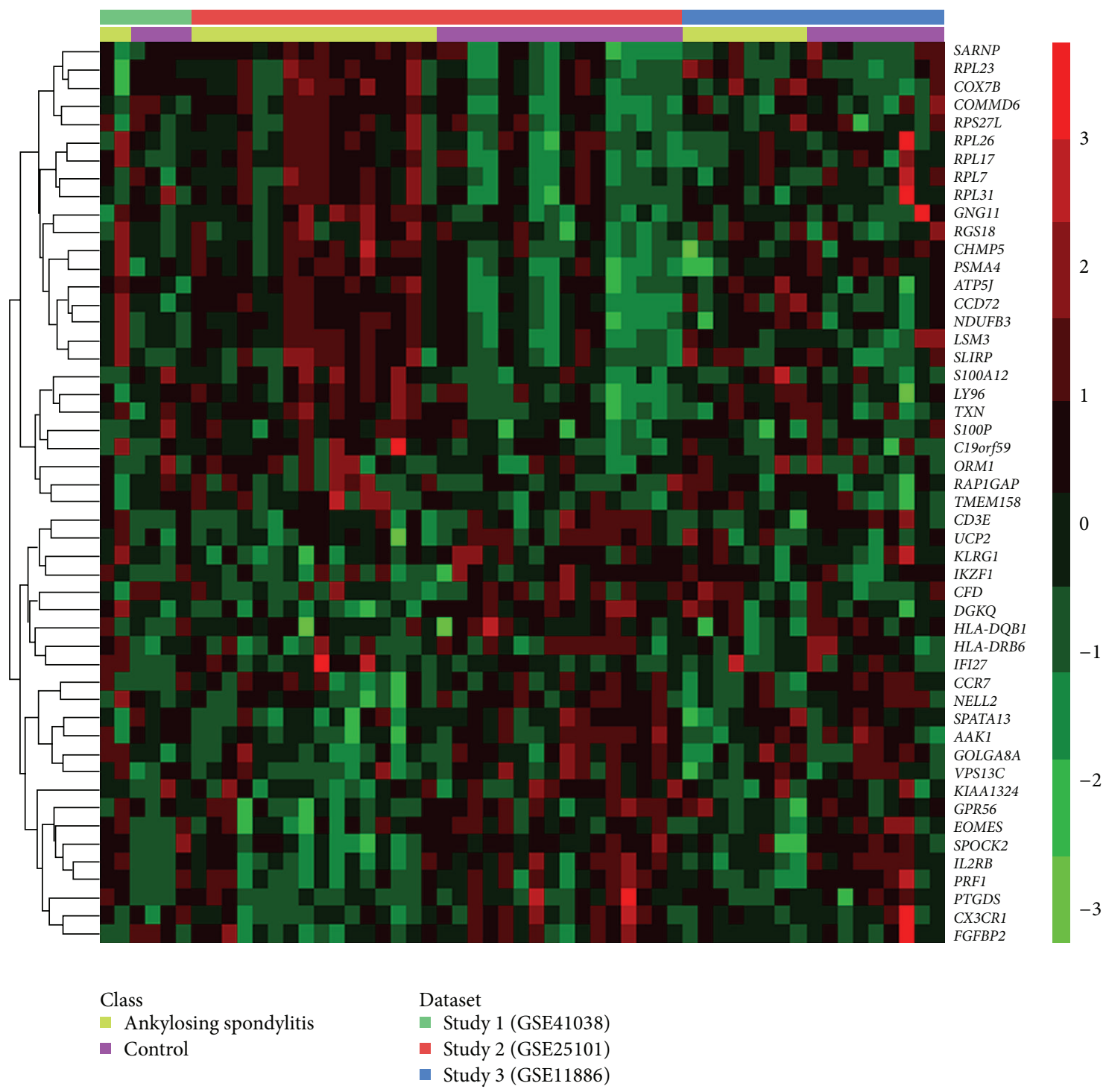

FIGURE 1: Heat map for visualization of gene pattern changes across different datasets (row-wise comparison). Individual datasets were rescaled in this map to prevent the influence of study-specific effects.

these results are suggestive rather than straightforward, those DE genes could be involved in the pathogenesis of ankylosing spondylitis and further research is required.

3.3. Advanced Analyses Results. Advanced analyses (GO analysis and pathway analysis) were carried out for further functional investigation of the DE genes. Figure 3 presented a summary of the GO analysis results. In the GO analysis, 715 GO terms were significantly enriched for the DE genes (see Supplementary Table 3), and the three most significantly enriched GO terms were "immune system process" $(P=3.46$ $\left.\times 10^{-26}\right)$, "immune response" $\left(P=7.83 \times 10^{-24}\right)$, and "defense response" $\left(P=6.99 \times 10^{-16}\right)$ (Table 3). In the pathway analysis, 15 significant pathways were identified when we mapped the DE genes to the KEGG database (see supplementary Table $4)$, and the three most significant pathways were antigen processing and presentation $\left(P=8.40 \times 10^{-5}\right)$, measles
$\left(P=3.30 \times 10^{-3}\right)$, and cell adhesion molecules (CAMs) $(P=$ $5.66 \times 10^{-3}$ ) (Table 4$)$.

\section{Discussion}

A number of genes have been reported to be upregulated or downregulated in ankylosing spondylitis patients [14-16]. Identification of the most important candidate genes and pathways involved in ankylosing spondylitis pathogenesis is a challenge currently. Growing high-throughput transcriptomic data enables meta-analysis of multiple datasets which has the likelihood of discovering robust candidates for diagnosis and treatment. Hence in this study, we performed a meta-analysis of multiple public microarray datasets to identify potential transcriptomic markers in developing ankylosing spondylitis.

In the meta-analysis, 905 consistently DE genes were identified in ankylosing spondylitis, among which 482 genes 
TABLE 2: The 10 most significantly upregulated and 10 most significantly downregulated genes in ankylosing spondylitis.

\begin{tabular}{|c|c|c|c|c|}
\hline Entrez ID & Gene symbol & Gene full name & $\begin{array}{c}\text { Combined rank } \\
\text { product }\end{array}$ & $\begin{array}{c}\text { Average log fold } \\
\text { change }\end{array}$ \\
\hline \multicolumn{5}{|c|}{10 most significantly upregulated genes } \\
\hline 2791 & GNG11 & Guanine nucleotide binding protein ( $\mathrm{G}$ protein), gamma 11 & 299.64 & 0.380467 \\
\hline 51372 & TMA7 & Translation machinery associated 7 homolog (S. cerevisiae) & 312.39 & 0.610581 \\
\hline 9349 & RPL23 & Ribosomal protein L23 & 324.13 & 0.148532 \\
\hline 23643 & LY96 & Lymphocyte antigen 96 & 330.33 & 0.195432 \\
\hline 170622 & COMMD6 & COMM domain containing 6 & 332.47 & 0.293187 \\
\hline 5909 & RAP1GAP & RAP1 GTPase activating protein & 343.66 & 0.223733 \\
\hline 1349 & COX7B & Cytochrome c oxidase subunit VIIb & 371.12 & 0.132367 \\
\hline 6283 & S100A12 & S100 calcium binding protein A12 & 439.12 & 0.334567 \\
\hline 27258 & LSM3 & LSM3 homolog, U6 small nuclear RNA associated (S. cerevisiae) & 440.15 & 0.596028 \\
\hline 199675 & C19orf59 & Chromosome 19 open reading frame 59 & 494.30 & 0.358146 \\
\hline \multicolumn{5}{|c|}{10 most significantly downregulated genes } \\
\hline 6286 & S100P & S100 calcium binding protein $\mathrm{P}$ & 335.94 & -0.199948 \\
\hline 3560 & $I L 2 R B$ & Interleukin 2 receptor, beta & 351.04 & -0.166370 \\
\hline 221178 & SPATA13 & Spermatogenesis associated 13 & 473.13 & -0.442046 \\
\hline 9289 & GPR56 & G protein-coupled receptor 56 & 528.50 & -0.239562 \\
\hline 22848 & $A A K 1$ & AP2 associated kinase 1 & 530.65 & -0.364595 \\
\hline 5551 & PRF1 & Perforin 1 (pore forming protein) & 531.02 & -0.114758 \\
\hline 1236 & CCR7 & Chemokine (C-C motif) receptor 7 & 609.87 & -0.199449 \\
\hline 1524 & CX3CR1 & Chemokine (C-X3-C motif) receptor 1 & 622.12 & -0.351950 \\
\hline 1675 & CFD & Complement factor D (adipsin) & 636.15 & -0.365854 \\
\hline 83888 & FGFBP2 & Fibroblast growth factor binding protein 2 & 653.39 & -1.054696 \\
\hline
\end{tabular}

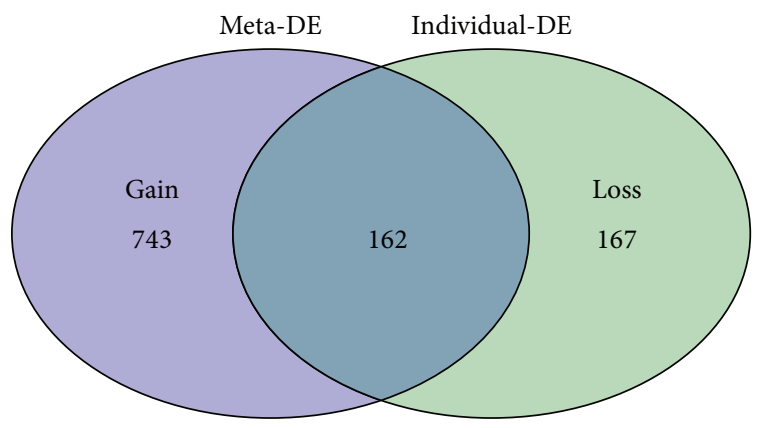

FIGURE 2: Venn diagram for overlap visualization between metaanalysis results and individual dataset analysis results. Meta-DE: DE genes identified in meta-analysis; individual-DE: DE genes identified in individual dataset analysis.

were upregulated and 423 genes were downregulated. The upregulated gene with the smallest combined RP was GNG11. GNG11 belongs to the G protein gamma family and plays a role in the transmembrane signaling system and cellular senescence $[11,12]$. The downregulated gene with the smallest combined RP was S100P. S100P encodes a protein which is a member of the S100 calcium-binding protein family and functions in the regulation of diverse cellular processes [13]. So far, S100P is reported to be involved in the development and progression of various cancers [13]. Although the exact contributions of these DE genes to ankylosing spondylitis

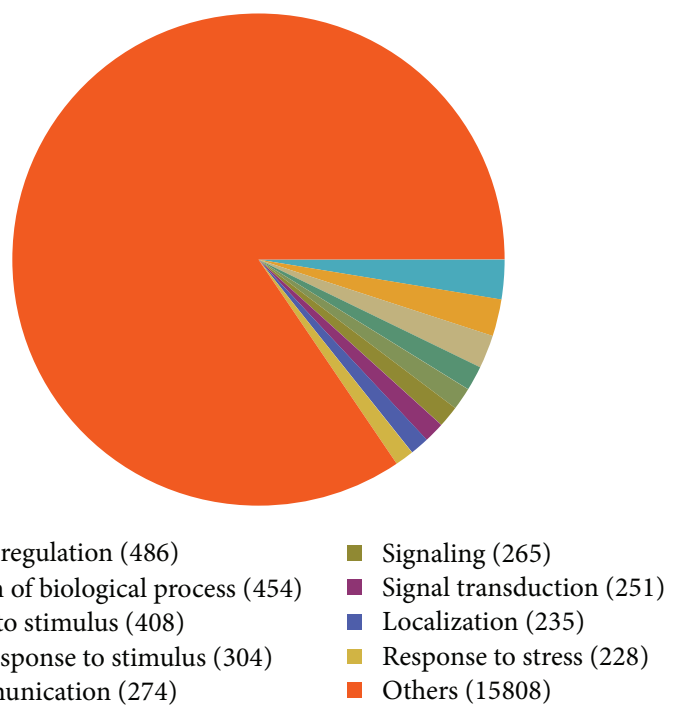

FIGURE 3: Summary of the GO analysis results for the DE genes in ankylosing spondylitis.

development are not clear yet, further research is necessary as those genes could be potential transcriptomic markers for ankylosing spondylitis.

Many consistently DE genes identified are involved in immune regulation, suggesting its major role in ankylosing spondylitis development. A set of DE genes without previous 
TABLE 3: The 10 most significantly enriched GO terms for the DE genes in ankylosing spondylitis.

\begin{tabular}{lcc}
\hline ID & Term & $P$ value \\
\hline GO:0002376 & Immune system process & $3.46 E-26$ \\
GO:0006955 & Immune response & $7.83 E-24$ \\
GO:0006952 & Defense response & $6.99 E-16$ \\
GO:0002682 & Regulation of immune system process & $3.01 E-13$ \\
GO:0006950 & Response to stress & $2.02 E-12$ \\
GO:0050776 & Regulation of immune response & $1.44 E-11$ \\
GO:0001775 & Cell activation & $2.24 E-11$ \\
GO:0045087 & Innate immune response & $5.19 E-11$ \\
GO:0045321 & Leukocyte activation & $1.32 E-10$ \\
GO:0030097 & Hemopoiesis & $2.91 E-10$ \\
\hline
\end{tabular}

TABLE 4: The 10 most significant pathways identified when the DE genes were mapped to the KEGG database.

\begin{tabular}{lc}
\hline Pathway & $P$ value \\
\hline Antigen processing and presentation & 0.0000840 \\
Measles & 0.0032959 \\
Cell adhesion molecules (CAMs) & 0.0056626 \\
Jak-STAT signaling pathway & 0.0070316 \\
Hypertrophic cardiomyopathy (HCM) & 0.0099861 \\
Pathogenic Escherichia coli infection & 0.0105380 \\
T cell receptor signaling pathway & 0.0167490 \\
Natural killer cell mediated cytotoxicity & 0.0172540 \\
Graft-versus-host disease & 0.0174410 \\
Allograft rejection & 0.0186970 \\
\hline
\end{tabular}

studies in ankylosing spondylitis were also identified in our analysis. Some of these DE genes may function in the pathogenesis of ankylosing spondylitis and could be potential biomarkers.

In the GO analysis, 715 significantly enriched GO terms were identified in total. The top three significantly enriched GO terms were "immune system process," "immune response," and "defense response." Similar features were also shown in a previous microarray analysis of ankylosing spondylitis mouse model [17]. The overlap of enriched GO terms between human study and mouse study suggests that immune response plays a major role in ankylosing spondylitis development and therefore deserves further studies. In the pathway analysis, 15 significant pathways were identified in all. The top three significant pathways were antigen processing and presentation, measles, and cell adhesion molecules (CAMs). Our result that pathways involved in immune response are related with the pathogenesis of ankylosing spondylitis is consistent with previous reports highlighting innate immune stimulation and the IL-23 pathway in ankylosing spondylitis pathogenesis $[18,19]$. Our analysis also identified pathways not previously studied in ankylosing spondylitis, some of which may harbor interesting functions and deserve further investigation.

In addition, all the results of our meta-analysis should be considered prudently due to the existence of several limitations. One limitation is the insufficient sample size used in our meta-analysis. A second limitation is the lack of subgroup analyses based on potential influential factors, including age, sex, treatment, disease severity, and platform usage, as ankylosing spondylitis is reported to be more prevalent in men and often occur in the third decade of life [1]. The third limitation is that biological knowledge base and pathway information are far from being complete at present and need further investigation. Hence, in order to achieve a more convincible conclusion, further analysis using larger sample size and more complete biological knowledge base and pathway information is required, and stratified analyses on different factors such as age, sex, disease severity, and platform usage are needed. In addition, experimental verification of the candidate $\mathrm{DE}$ genes identified should also be performed in the future, and functional studies need to be carried out as well to address the exact roles of those candidate $\mathrm{DE}$ genes in ankylosing spondylitis.

In conclusion, we identified consistently $\mathrm{DE}$ genes in ankylosing spondylitis that could potentially serve as transcriptomic markers. GO and pathway analyses revealed that those candidates strongly were associated with immune system process besides the underlying complex and multifactorinfluenced molecular mechanism. These results provide novel insights into the pathogenesis of ankylosing spondylitis and promote the generation of diagnostic gene sets.

\section{Conflict of Interests}

The authors declare that there is no conflict of interests regarding the publication of this paper.

\section{Acknowledgments}

This work was supported by grants from key medical subjects of Jiangsu Province (Grant no. XK201120); Innovative Team of Jiangsu Province (Grant no. LJ201114); Special Clinical Medical Science and Technology of Jiangsu Province (Grant nos. BL2012050 and BL2013014); Key Laboratory of Suzhou (Grant nos. SZS201108 and SZS201307); and National Natural Science Foundation (Grant nos. 81100371, 81370627, 81300423, and 81272143).

\section{References}

[1] A. El Maghraoui, "Extra-articular manifestations of ankylosing spondylitis: prevalence, characteristics and therapeutic implications," European Journal of Internal Medicine, vol. 22, no. 6, pp. 554-560, 2011.

[2] L. E. Dean, G. T. Jones, A. G. Macdonald, C. Downham, R. D. Sturrock, and G. J. Macfarlane, "Global prevalence of ankylosing spondylitis," Rheumatology, vol. 53, no. 4, pp. 650-657, 2014.

[3] F. M. Pimentel-Santos, D. Ligeiro, M. Matos et al., "Whole blood transcriptional profiling in ankylosing spondylitis identifies novel candidate genes that might contribute to the inflammatory and tissue-destructive disease aspects," Arthritis Research and Therapy, vol. 13, Supplement, no. 2, article no. R57, 2011. 
[4] J. A. Smith, M. D. Barnes, D. Hong, M. L. DeLay, R. D. Inman, and R. A. Colbert, "Gene expression analysis of macrophages derived from ankylosing spondylitis patients reveals interferon$\gamma$ dysregulation," Arthritis and Rheumatism, vol. 58, no. 6, pp. 1640-1649, 2008.

[5] J. Xia, C. D. Fjell, M. L. Mayer, O. M. Pena, D. S. Wishart, and R. E. W. Hancock, "INMEX-a web-based tool for integrative meta-analysis of expression data," Nucleic Acids Research, vol. 41, pp. W63-70, 2013.

[6] F. Hong, R. Breitling, C. W. McEntee, B. S. Wittner, J. L. Nemhauser, and J. Chory, "RankProd: a bioconductor package for detecting differentially expressed genes in meta-analysis," Bioinformatics, vol. 22, no. 22, pp. 2825-2827, 2006.

[7] T. Barrett, S. E. Wilhite, P. Ledoux et al., "NCBI GEO: archive for functional genomics data sets-update," Nucleic Acids Research, vol. 41, no. 1, pp. D991-D995, 2013.

[8] S. van der Linden, H. A. Valkenburg, and A. Cats, "Evaluation of diagnostic criteria for ankylosing spondylitis. A proposal for modification of the New York criteria," Arthritis and Rheumatism, vol. 27, no. 4, pp. 361-368, 1984.

[9] S. Falcon and R. Gentleman, "Using GOstats to test gene lists for GO term association," Bioinformatics, vol. 23, no. 2, pp. 257-258, 2007.

[10] M. Kanehisa and S. Goto, "KEGG: kyoto encyclopedia of genes and genomes," Nucleic Acids Research, vol. 28, no. 1, pp. 27-30, 2000.

[11] M. N. Hossain, R. Sakemura, M. Fujii, and D. Ayusawa, "Gprotein $\gamma$ subunit GNG11 strongly regulates cellular senescence," Biochemical and Biophysical Research Communications, vol. 351, no. 3, pp. 645-650, 2006.

[12] E. A. Balcueva, Q. Wang, H. Hughes, C. Kunsch, Z. Yu, and J. D. Robishaw, "Human G protein $\gamma_{11}$ and $\gamma_{14}$ subtypes define a new functional subclass," Experimental Cell Research, vol. 257, no. 2, pp. 310-319, 2000.

[13] V. Tóthová and A. Gibadulinová, "S100P, a peculiar member of S100 family of calcium-binding proteins implicated in cancer," Acta Virologica, vol. 57, no. 2, pp. 238-246, 2013.

[14] R. Fischer, D. C. Trudgian, C. Wright et al., "Discovery of candidate serum proteomic and metabolomic biomarkers in ankylosing spondylitis," Molecular \& cellular proteomics: $M C P$, vol. 11, no. 2, 2012.

[15] C. Romero-Sanchez, H.-K. Tsou, M.-S. Jan et al., "Serum monocyte chemotactic protein-1 concentrations distinguish patients with ankylosing spondylitis from patients with mechanical low back pain," Journal of Spinal Disorders \& Techniques, vol. 24, no. 3, pp. 202-207, 2011.

[16] D. L. Mattey, J. C. Packham, N. B. Nixon et al., "Association of cytokine and matrix metalloproteinase profiles with disease activity and function in ankylosing spondylitis," Arthritis Research and Therapy, vol. 14, no. 3, article R127, 2012.

[17] K. R. Haynes, A. R. Pettit, R. Duan et al., "Excessive bone formation in a mouse model of ankylosing spondylitis is associated with decreases in Wnt pathway inhibitors," Arthritis Research and Therapy, vol. 14, no. 6, article R253, 2012.

[18] P. C. Robinson and M. A. Brown, "Genetics of ankylosing spondylitis," Molecular Immunology, vol. 57, no. 1, pp. 2-11, 2014.

[19] A. Cortes, J. Hadler, J. P. Pointon et al., "Identification of multiple risk variants for ankylosing spondylitis through highdensity genotyping of immune-related loci," Nature Genetics, vol. 45 , no. 7, pp. 730-738, 2013. 


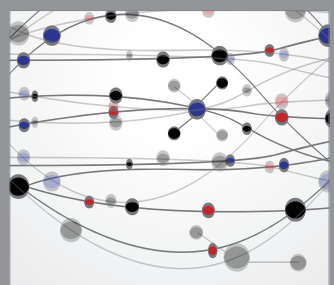

The Scientific World Journal
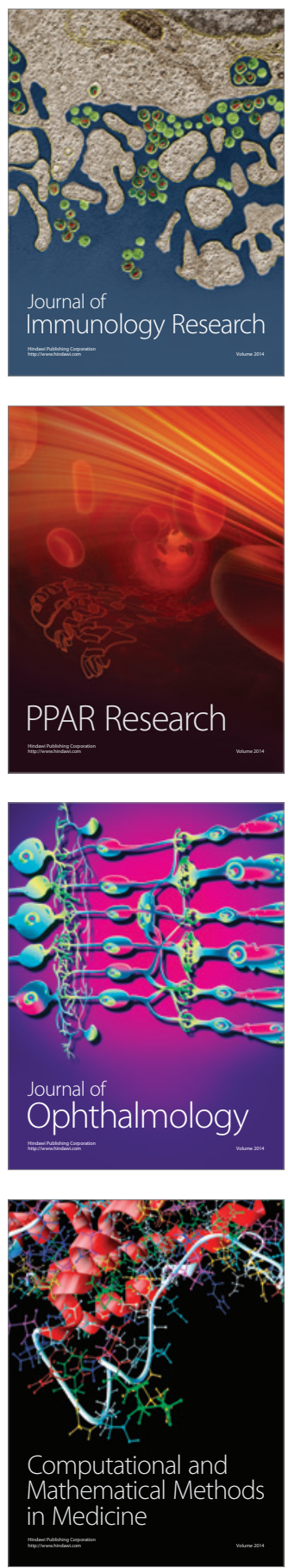

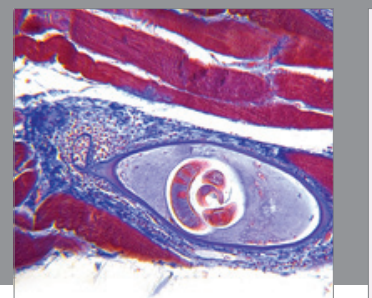

Gastroenterology

Research and Practice
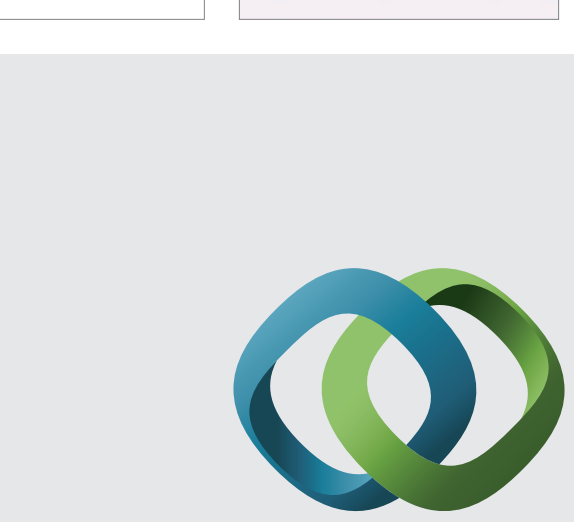

\section{Hindawi}

Submit your manuscripts at

http://www.hindawi.com
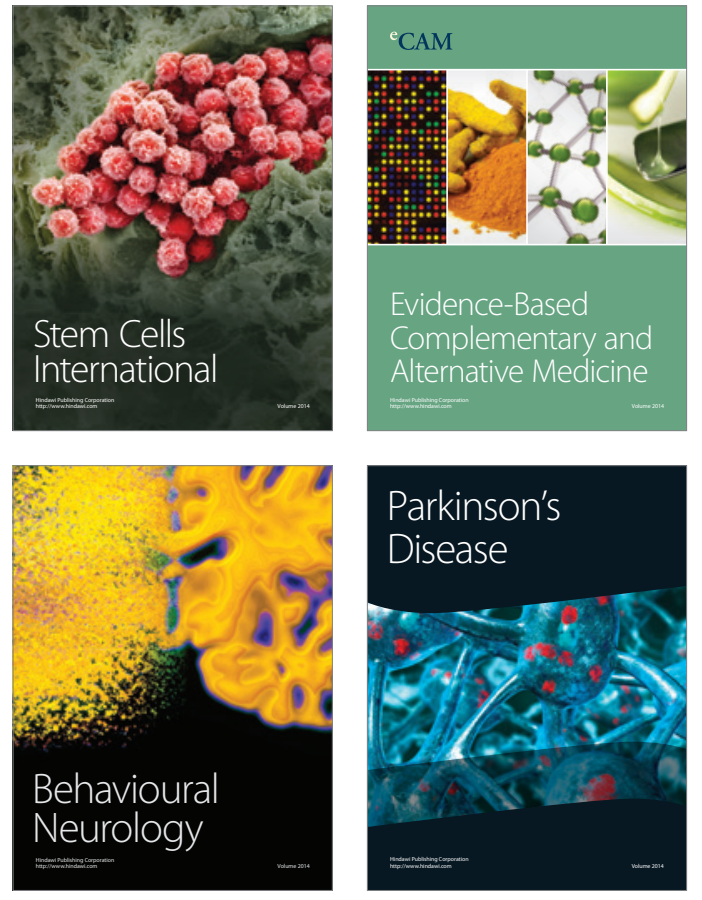
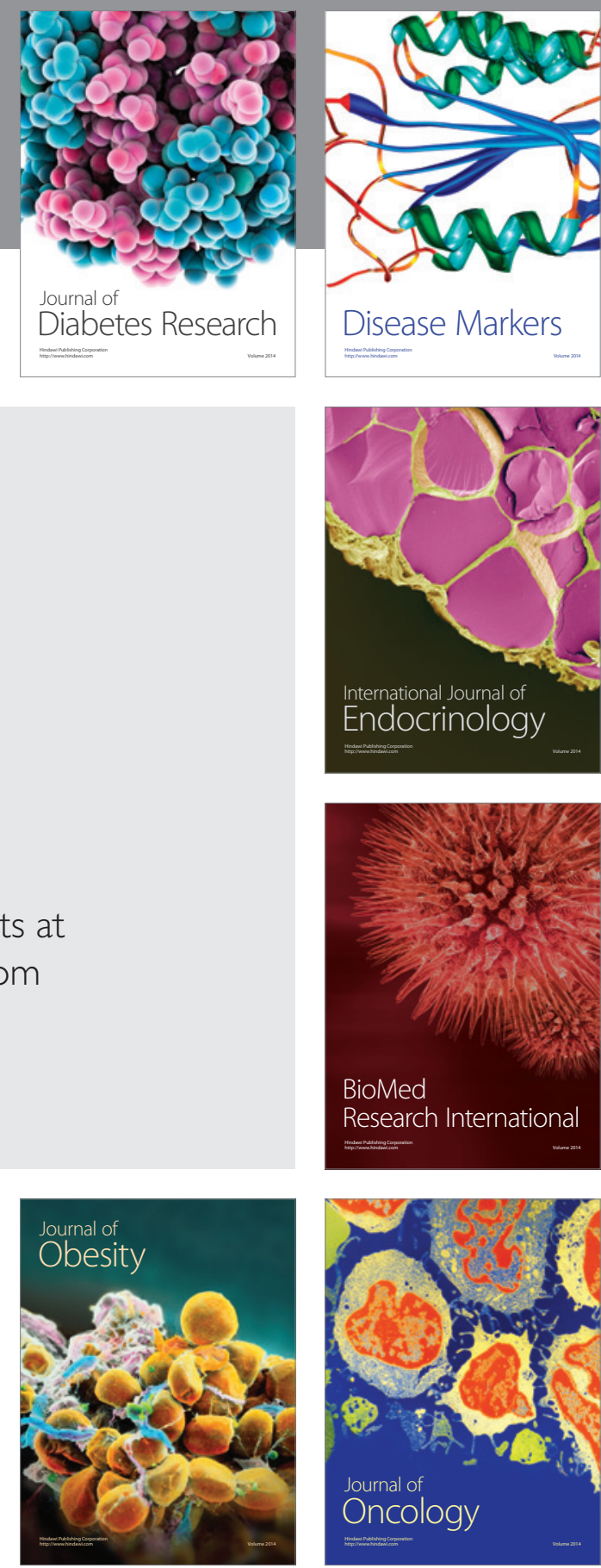

Disease Markers
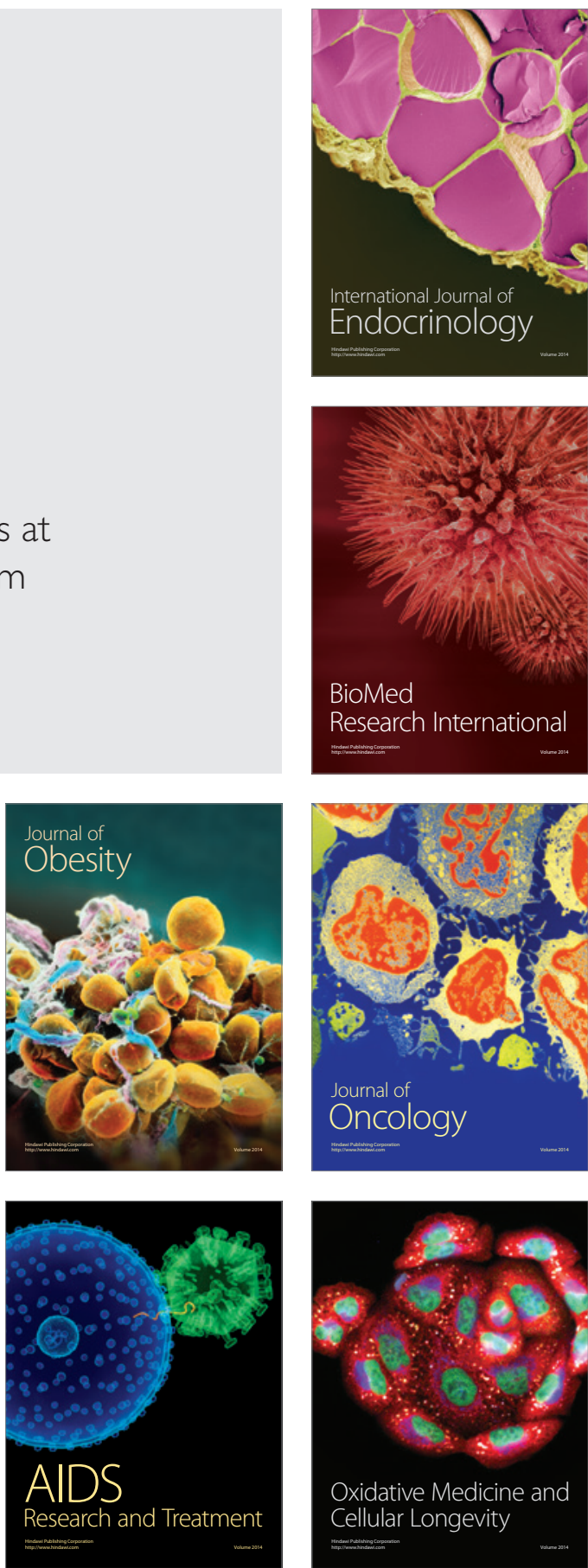